\title{
TWO-PHASE FLOW MODELLING IN GAS-STIRRED LIQUID VESSELS WITH SUPG-STABILIZED EQUAL-ORDER INTERPOLATIONS
}

\author{
N. NIGRO, M. STORTI AND S. IDELSOHN \\ Grupo de Tecnologia Mecánica del INTEC, Universidad Nacional del Liboral and CONICET, Güemes 3450, 3000 \\ Santa Fe, Argentina
}

\begin{abstract}
SUMMARY
The modelling of liquid flow in gas-stirred vessels is described. A simple two-phase model accounts for the buoyancy effect of bubbles. Friction between liquid and gas is modelled with the hypothesis of independent bubbles. The resulting PDE system is discretized with an original version of the SUPG-FEM technique which stabilizes both the convection term and equal-order interpolations for velocity and pressure, which are known to be unstable for incompressible flows. The resulting steady state discrete system is solved via pseudotemporal explicit iteration with a local time step and a preconditioning to homogenize the temporal scales for liquid and gas.
\end{abstract}

KEY wORDS Ladle Two-phase flow Navier-Stokes SUPG Streamline diffusion Stabilized algorithm

\section{INTRODUCTION}

The continuous casting process is depicted in Figure 1. Molten material is homogenized in both temperature and concentration in the ladle furnace. Once this homogenization is achieved, the content of the furnace is spilled into the tundish, which in turn feeds several continuous casting moulds. It is very important to keep the temperature with a narrow gap and this is hindered by thermal losses at each stage of the process. The metal in the ladle is stirred by injection of an inert gas, e.g. argon, to promote homogenization.

In the present work we describe an algorithm to model such vessels, with special attention to the determination of the velocity field. Discretization is based on the SUPG (streamline upwind Petrov-Galerkin) version of the FEM (finite element method). The SUPG concept is used to stabilize not only the advection term but also the sonic operator, whose complex structure (it is a non-diagonalizable system) is responsible for the incompressibility condition. A stabilizing term proportional to the Laplacian of pressure in the continuity equation and another identical to a bulk viscosity one are generated in a natural way, keeping the consistency unaltered (SUPG is a weighted residual technique). The nature of these stabilizing terms is studied in depth for the simpler Stokes $(R e=0)$ equations.

\section{TWO-PHASE MODEL}

The balance equations for each phase are

$$
(\partial(\rho \psi) / \partial t+\nabla \cdot(\rho \mathrm{u} \psi))=\rho \phi-\nabla \cdot \mathbf{J},
$$

CCC 0271-2091/94/130001-22

Received 3 March 1993

(C) 1994 by John Wiley \& Sons, Ltd.

Revised 7 December 1993 


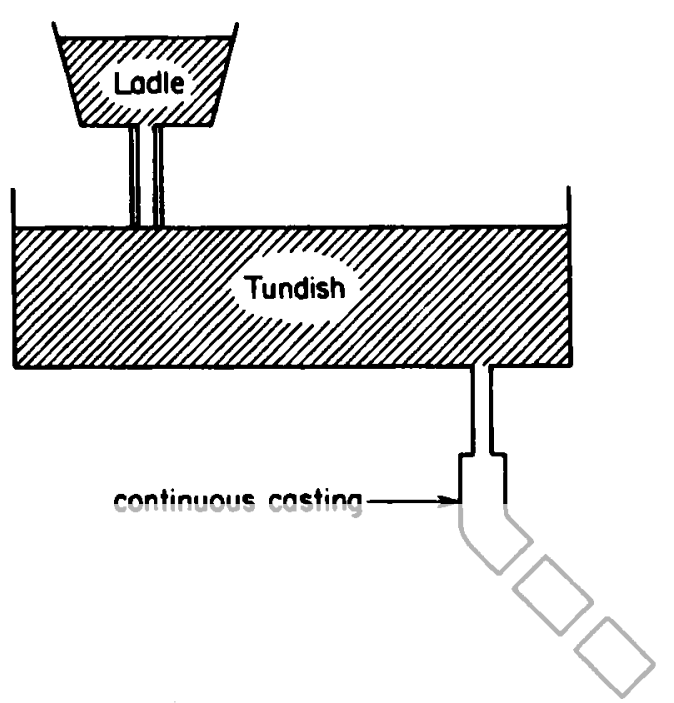

Figure 1. Schematic description of the continuous casting process
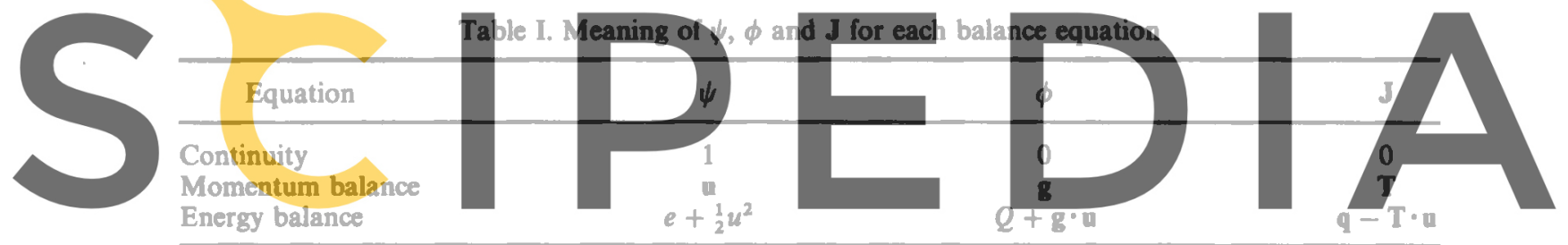

Register for free at https//www.scipedia.com to download the version without the watermark

where $\psi$ is a fuid property, a $\psi$ is the convective fux, $\phi$ is a bulk source term and $J$ is the difisive nux. The corresponding quantities for each of the continuity, momentum and energy equations are given in Table $\mathbf{I}$, where $T$ is the stress tensor (including the isotropic component), $\mathbf{q}$ is the heat flux vector, $\mathbf{g}=\boldsymbol{g} \hat{\mathbf{k}}$ the gravity vector, $\hat{\mathbf{k}}$ is a unit vector along the $z$-axis and $Q$ is a heat source per unit volume.

Applying the volume-averaging operator ${ }^{1,2}$ to that part of the volume instantaneously occupied by each phase, we arrive at the expressions

$$
\begin{gathered}
\partial\left(\alpha_{k}\langle\rho\rangle_{k}\right) / \partial t+\nabla\left(\alpha_{k}\langle\rho \mathbf{u}\rangle_{k}\right)=0, \\
\partial\left(\alpha_{k}\langle\rho \mathbf{u}\rangle_{k}\right) / \partial t+\nabla\left[\alpha_{k}\langle(\rho u \mathbf{u}-\mathbf{T})\rangle_{k}\right]=\alpha_{k}\langle\rho\rangle_{k} \mathbf{g} \pm \mathbf{F}_{\mathrm{GL}},
\end{gathered}
$$

where $\alpha_{k}$ is the volume fraction occupied by phase $k$ and \langle\rangle$_{k}$ is the average operator over it. For the liquid $\rho$ is constant and we assume that space correlation factors are unity, e.g. $\langle u u\rangle_{L}=\langle u\rangle_{L}\langle u\rangle_{L}$. We obtain a system of equations similar to the Navier-Stokes one with an additional source term in the momentum equations given by

$$
\begin{aligned}
\mathbf{F}_{\mathbf{G L}} & =\frac{1}{\Omega} \times(\text { number of bubbles in } \Omega) \times(\text { force over one isolated bubble) } \\
& =\frac{1}{\Omega} \times \frac{\Omega_{\mathrm{G}}}{\boldsymbol{\Omega}_{\mathrm{b}}} \times 4 \pi \mu\left(\mathrm{u}_{\mathrm{G}}-\mathrm{u}_{\mathrm{L}}\right) r_{\mathrm{b}} F^{*}\left(R e_{\mathrm{b}}\right)
\end{aligned}
$$


where $R e_{b}=\left|u_{G}-u_{L}\right| r_{b} / v$ is the bubble Reynolds number and $r_{b}=\left(3 \Omega_{b} / 4 \pi\right)^{1 / 3}$ is the bubble radius or the equivalent one if it is not spherical. From all the forces acting on the bubble $e^{3.4}$ we have retained the drag force only. Thus we neglect the virtual mass, Baxten and Magnus forces. Perhaps the most important among them is the virtual mass one, but we neglect it since the flow is driven mainly by the high ferrostatic gradient of pressure. The bubble radius is a function of pressure and temperature given by the ideal gas law, with a prescribed value at the inlet nozzle. The variation in the bubble radius is mostly due to the ferrostatic variation in the pressure, which is several atmospheres higher at the bottom of the ladle than at the top for common dimensions. $F^{*}$ is the non-dimensional drag (with respect to Stokes flow) on an isolated bubble: $F^{*} \rightarrow 1$ as $R e_{b} \rightarrow 0$. For the force on an isolated spherical gaseous bubble we have taken the Stokes formula corrected for the case where the sphere is not solid but is a fluid of viscosity $\mu^{\prime}$. The corrected expression for the drag is ${ }^{5} F=2 \pi\left(u_{L}-u_{G}\right) \mu r_{b}\left(2 \mu+3 \mu^{\prime}\right) /\left(\mu+\mu^{\prime}\right)$. In the limit of a gaseous bubble, $\mu^{\prime} / \mu \rightarrow 0$, the expression gives $F=4 \pi\left(n_{T}-n_{G}\right) \mu r_{b}$, i.e two-thirds of the classical result for solid spheres $\left(\mu^{\prime} \rightarrow \infty\right)$.

Finally, the friction force must vanish when one of the phases disappears. This is so when the gas is absent owing to the $\alpha_{G}$-factor, but it is not when $\alpha_{\mathrm{L}}=0$. In consequence, we add a factor $1-\alpha_{G}$ in the friction term (4). To some extent this term takes account the bubble coalescence when the gas volume fraction becomes noticeable. The final expression is
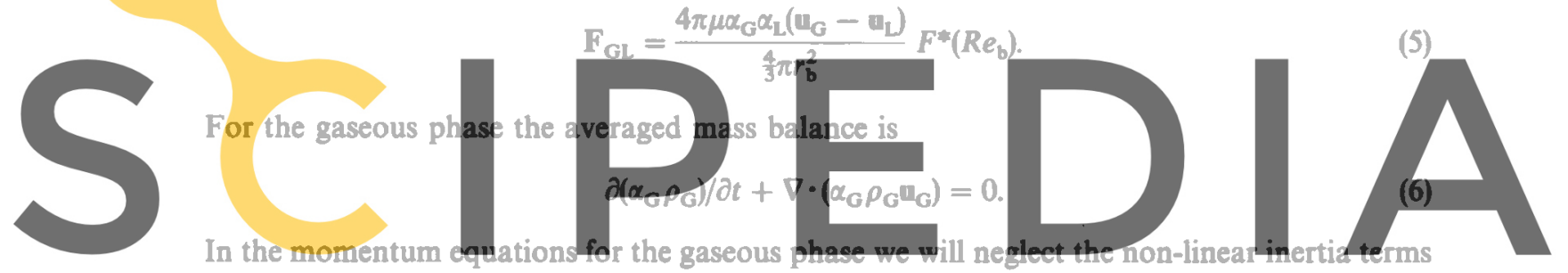

In the momentum dquations for the gaseous phase we will neglect the non-linearinertid terms

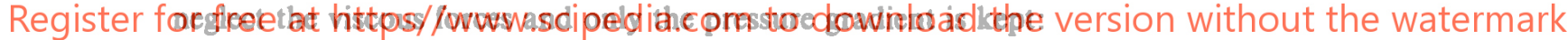

$$
\nabla\left(\alpha_{G} p\right)-\alpha_{\mathrm{G}} \rho_{\mathrm{G} g}-\mathbb{F}_{\mathrm{GL}}=0,
$$

where $p$ is the pressure, which is assumed to be the same for both phases. This last expression allows us to compute the local 'slip' velocity between the phases as

$$
u_{G}-u_{L}=\frac{\frac{1}{3} r_{b}^{2}}{\mu \alpha_{G} \alpha_{L} F^{*}\left(R e_{b}\right)}\left[\nabla\left(\alpha_{G} p\right)-\alpha_{G} \rho_{G} g\right] \text {. }
$$

Equations (6) for the gas volume fraction together with the Navier-Stokes equations for the liquid with the friction term and appropriate boundary conditions form a closed system. The resulting model can be classified as a two-fluid model where the equation for the gas is simply an advection equation.

\section{NUMERICAL APPROXIMATION}

The resulting system of PDEs (mass, momentum, energy balance for the liquid and mass balance for the gas) is an incompletely parabolic one. Two main difficulties arise when standard numerical methods such as the FEM, FDM, or FVM are applied to it, namely the incompressibility restriction and the advective character at high Reynolds numbers based on cell size. The first is associated with low Mach numbers, being defined as the ratio between the local velocity of 
the fluid and the local speed of sound. Highly incompressible fluids such as water and most liquids exhibit incompressible flow patterns (low Mach numbers) in most common situations. Surprisingly enough, gases, which are much more compressible, exhibit incompressible flow patterns if the characteristic velocity is low with respect to the speed of sound. Furthermore, flows at high Mach numbers can exhibit local incompressible behaviour at stagnation points. When centred equal-order methods are applied to flows with incompressible behaviour, spurious oscillations in pressure (also called checker-board modes) are obtained in regions with high gradients, e.g. singularities, stagnation points and corners. By centred equal-order methods we mean, in an FEM context, the Galerkin formulation with the same degree of polynomial interpolation for velocities and pressures, e.g. bilinear continuous velocities and bilinear discontinuous pressure (Q1/Q1 element) for quadrangles (see Figure 2). It has been found that lowering the order of interpolation for pressure by one stabilizes the interpolation in some cases (Q2/P1 element) but is not sufficient in others (P1/P0 and $\mathrm{P} 2 / \mathrm{P} 1$ for triangles, $\mathrm{Q} 1 / \mathrm{P} 0, \mathrm{Q}^{-} / \mathrm{P} 1$ and $\mathrm{Q} 2 / \mathrm{Q} 1$ for quadrangles). ( $\mathrm{P} n$ stands for interpolations (discontinuous or not) which retain all terms in polynomials in space variables up to order $n ; \mathrm{P} 1$ and P2 stand for linear and quadratic respectively. Qn stands for tensor products of polynomials in each variable up to $n$; Q2 $2^{-}$stands for the serendipity (eight nodes) interpolation.) Some of these can be stabilized by further lowering the interpolation order for pressure, e.g. P2/P0 and $\mathrm{Q}^{-} / \mathrm{P} 0$, but they do not yield optimal rates of convergence. An interesting way to stabilize approximations is to enlarge the velocity space through the addition of bubble functions, which are higher-order functions that vanish on the element boundary (the element $\mathrm{P}^{+} / \mathrm{P} 1$ for instance). In fact; it seems that any interpolation can be stabilized through the addition of a large enough number of bubble functions. All the previous discussion was based on discontinuous interpolations since they are the most familiar to FEM users, but something similar applies continuous interpolations for pressure. The correct setting for und is a stability condition that must be satisfied by the pair of interpolation spaces, calfed the Brezzi-Babǔska condition. This condition is common to all problems where internal constraints

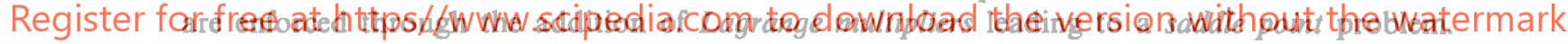

Other physical situations where this condition arises are incompressible elasticity, plate theory and viscoelasticity. In the context of the FDM equal-order interpolation is the equivalent of non-staggered grids and stable approximations are obtained through the use of staggered grids.
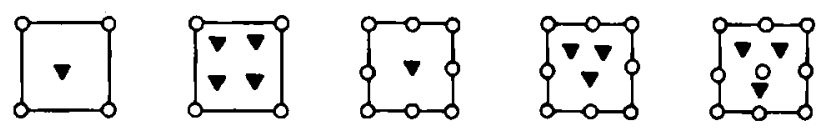

Q1/PO

Q1/Q1

Q2/PO

Q2'/P1

Q2/P1
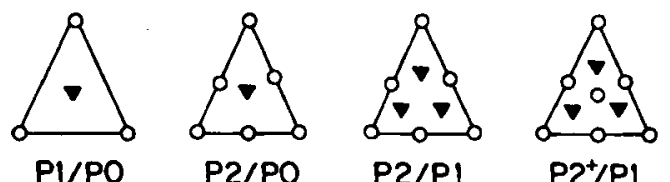

- velocity node

$\checkmark$ pressure node

Figure 2. Family of finite element interpolation spaces with discontinuous interpolation for pressure. Only the $\mathbf{Q 2}^{-} / \mathrm{PO}$ and $\mathrm{Q} 2 / \mathrm{P} 1$ interpolations are stable for quadrangles and the $\mathrm{P} 2 / \mathrm{P} 0$ and $\mathrm{P2}^{+} / \mathrm{P} 1$ for triangles 


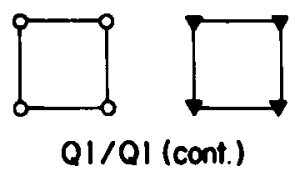

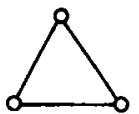

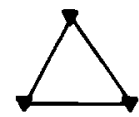

PI /PI (cont.)

- velocity node

$\checkmark$ pressure node

Figure 3. Family of finite element interpolation spaces with continuous interpolation for pressure. Both interpolations are unstable

The implementation of stable FEM approximations is particularly hard in 3D. In some cases they involve bubble functions at the faces and in general are difficult to code. Another possibility is to use equal-order interpolations stabilized through the addition of terms in the variational formulation. In this way very simple interpolation patterns, e.g. P1/P1 triangles, Q1/Q1 quadrangles (see Figure 3) and their natural extensions to 3D, can be safely used. It is worth stressing the fact that the stabilizing terms do not break the consistency, i.e. the stabilized formulation is a weighted residual one. Moreover, it has been shown that under certain restrictions the stabilizing terns are equivalen: to the addition of certais not a priori known, bubble functions. One interesting fact about stabilized formulations is that they seem to be stable in much broader situations than those for which they have ben devised, unlike the "a pripri' ones. For instance, the Q2/P1 and Q1/P0 interpolations seem to be unstable under the addition of the Coriolis term arising in turbomachinery and mechanically stirred reactors, whereas the stabilized algorithms have proved to be stable. The second source of instability mentioned at the beginning of this section, namely the pure advective character arising at high Reynolds

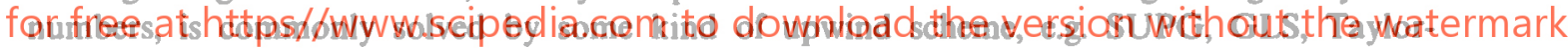
Galerkin, etc. The algorithm we present here stabilizes both sources of instability at the same time.

The addition of a gaseous bubbly phase greatly increases the compressibility of the mixture, even for small concentrations of gas. On the other hand, the density is not very much affected by small concentrations of gas, so that it is expected that the incompressible character will be lost in regions where the concentration of gas is relevant. However, there always exist regions where the concentration of gas is strictly zero and thus where the fluid behaves as incompressible.

The rest of the section will be devoted to the stabilized algorithm we propose. The numerical discretization is based on a weighted residual Petrov-Galerkin method, which means that the weight functions are not necessarily equal to the interpolation ones and, furthermore, are allowed to be discontinuous across inter-element boundaries. The particular choice of weight functions according to the SUPG method adds numerical diffusion to the discrete system, thus suppressing the spurious oscillations which are typically encountered when centred schemes are applied to advection-dominated problems.

We followed this line further: the SUPG method is used to stabilize equal-order interpolations, which, as it is well known, suffer spurious oscillations in pressure for incompressible flows. Stabilizing terms similar to those obtained by other authors are obtained in a natural way. The method is discussed in detail in the following subsections. In Section 3.1 the SUPG method as applied to the 1D scalar advection-diffusion equation is recalled. In Section 3.2 the natural extension to systems is introduced. In Section 3.3 these results are extended to several spatial 
dimensions. In Section 3.4 the method is applied to the Stokes system, while an in-depth analysis of the stability terms involved is given in Section 3.5 .

\subsection{One-dimensional scalar advection-diffusion equation}

The equation to be solved is

$$
a u_{, x}=k u_{, x x},
$$

where $a$ is the advection velocity for the variable $u$ and $k$ is the diffusion. As is well known, centred finite difference discretizations are unstable, i.e. the resulting discrete field suffers from spurious oscillations. This problem can be solved if the diffusivity $k$ in the discrete system is replaced by $k^{\prime}=k+k^{\text {num }}$, with

$$
k^{\mathrm{num}}=a^{2} \tau
$$

where

$$
\tau=\frac{\Delta x}{2|a|} \psi(P e), \quad \psi(P e)=\operatorname{coth}\left(\frac{P e}{2}\right)-\frac{2}{P e}, \quad P e=\frac{a \Delta x}{k} .
$$

Here $\tau$ is a parameter with the dimension of time, called the intrinsic time, and $\psi$ is the so-called 'magic' function (see Figure 4). In a finite element context this scheme can be obtained by modifying the test functions in the form $W_{i}(x)=N_{i}(x)+\tau a N_{i, x}$, where $N$ is the interpolation function corresponding to node $i$ and $W_{i}$ is the corresponding test function.

The resulting schene gives exact values for constant coefficient problems without a source term, discretized on hornogeneous grids.

\subsection{One-dimensional advective-diffusion systems}

Register for

$$
\mathbf{A U}, x=\mathbb{K} \mathbf{U}_{, x x}, \quad \mathbf{U} \in \mathbb{R}^{m}, \quad \mathbb{A}, \mathbb{K} \in \mathbb{R}^{m \times m} .
$$

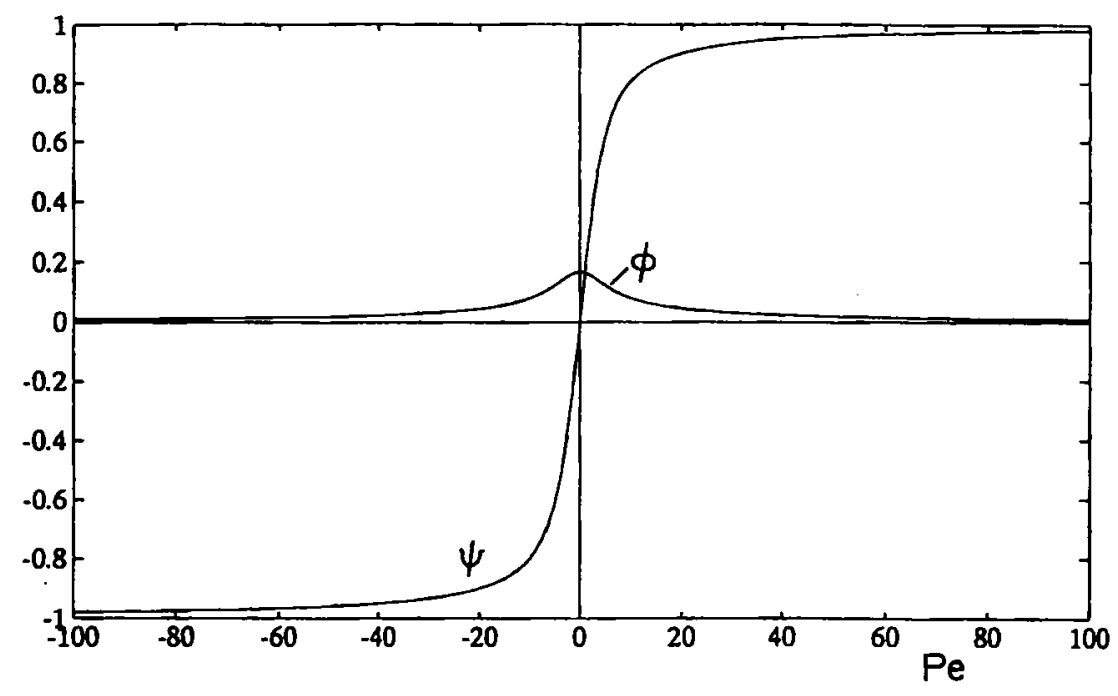

Figure 4. Magic function $\psi(P e)$ and its associated $\phi$ 
Multiplying this equation by $\mathbf{K}^{-1}$ and transforming into new variables $\mathbf{V}=\mathbf{S}^{-1} \mathbf{U}$, where $\mathbf{S}$ is the matrix which solves the eigenvalue problem

$$
\mathbf{S}^{-1} \mathbf{K}^{-1} \mathbf{A S}=\Lambda,
$$

with $\Lambda$ a diagonal matrix, the system (12) decouples into $m$ independent advection-diffusion equations. These equations are solved as in the previous subsection and, reverting to the U-variables, the discretized system is

$$
A \frac{\mathbf{U}_{i+1}-\mathbf{U}_{i-1}}{2 \Delta \mathbf{x}}=\left(\mathbf{K}+\mathbf{K}^{\text {num }}\right) \frac{\mathbf{U}_{i+1}-2 \mathbf{U}_{i}+\mathbf{U}_{i-1}}{\Delta x^{2}},
$$

where

$$
\mathbb{K}^{\text {num }}=\mathbf{A} \tau \mathbf{A}, \quad \tau=\frac{\Delta x^{2}}{2} \phi\left(\mathbb{K}^{-1} \mathbf{A} \Delta x\right) \mathbf{K}^{-1}
$$

and $\phi$ is a real-valued function defined as $\phi(x)=\psi(x) / x$ (see Figure 4). As usual, the evaluation of the function $\phi$ on its matricial argument is carried out by solving an eigensystem decomposition of the matrix and evaluating the function on its eigenvalues. This scheme also gives exact values under similar conditions as specified for the scalar case at the end of the previous subsection. We stress the fact that this result is new as far as we know, whereas the scheme for the scalar case is a wll-established one. 3.3. Multidimensional advective-diffusioe scalar equations and systems

For multidimensional problems the key point is to add numerical diffusion direction of the local advection vector. This is done by the following definition of nunerical diffusion:

Register for free at httpsh/www, scipedia,com to download the version without the watermark

This expression is valid for orthogonal grids. For non-orthogonal grids a transformation to the master element is performed. ${ }^{6}$ (This is equivalent to the transformation to grid co-ordinates in the FDM.)

Expression (16) can be written in the advection-dominated limit $(P e \rightarrow \infty)$ as (for simplicity we consider an orthogonal, square grid)

$$
\tau^{-2}=\tau_{x}^{-2}+\tau_{y}^{-2}+\tau_{z}^{-2}, \quad \tau_{x}=\Delta x / 2\left|a_{x}\right|,
$$

which can be regarded as a composition of critical times in stability analysis. Then the extension to multidimensional advective-diffusive systems we propose is

$$
\mathbf{K}_{i j}^{\text {num }}=\mathbf{A}_{i} \tau \mathbf{A}_{j}
$$

with

$$
\tau^{-p}=\tau_{x}^{-p}+\tau_{y}^{-p}+\tau_{z}^{-p}
$$

where

$$
\tau_{i}=\left(\Delta x_{i}^{2} / 2\right) \phi\left(\mathbf{K}_{i i}^{-1} \mathbf{A}_{i} \Delta x_{i}\right) \mathbf{K}_{i i}^{-1}, \quad i=1,2,3,
$$

and $p$ is a positive number. 
It can be shown that in the advection-dominated limit equation (20) reduces to the scheme proposed by Mallet, ${ }^{7}$ the only difference being that the absolute value of the jacobian matrices must be interpreted in the metrics of $\mathbf{K}_{i i}$. Both schemes are equivalent in the following sense: they give a set of $m$ uncoupled, fully upwinded equations for the eigencomponents but on a different basis.

\subsection{Application to the Stokes equations}

When the Reynolds number is very low, the convection term in the Navier-Stokes equations, coming from the inertia in the momentum equations, can be neglected and we obtain the Stokes equations. In what follows we will express the Stokes system as an advective-diffusive one. This could seem contradictory, since the convection term has been neglected and it could be thought that the remaining set of equations is purely diffusive, but here 'advective' must be understood in a general sense. If some perturbation is added to a steady state of a compressible viscous fluid, the perturbation is decomposed into a series of waves which are damped as they move through the fluid and partially absorbed or reflected when they reach the boundary. One component is the vorticity wave, which travels with the velocity of the fluid and is damped according to the Reynolds number. This is the analogue of shear (transverse) waves in elastic solids. On the other hand, pressure waves propagates at the speed of sound in all directions and are the analogue of compression (longitudinal) waves in solids. For a fluid at rest, vorticity waves can only be damped, but pressure waves still exist and are responsible for the enforcement of the incompressibility condition. Here 'adyective' also stands for those terns responsible for the propagation of the pressure waves.

In what follows we will show what kind of scheme is obtained for the Stokes equations and will compare it with other types of stabilization techniques. The Stokes equations we are considering are

$$
\nabla \cdot \mathbf{u}=0, \quad \nabla p-v \Delta \mathbf{u}=\mathbf{f} .
$$

Register for free at https//www.scipedia.com to download the version without the watermark This system can be put in the context of homogenous linear advective-diffusive systems through the following definitions of the flux Jacobians:

$$
\mathbf{U}=\left[\begin{array}{l}
\mathbf{u} \\
p
\end{array}\right], \quad \mathbf{A}_{j} k_{j}=\left[\begin{array}{cc}
\mathbf{0} & \mathbf{k} \\
\mathbf{k}^{\mathrm{T}} & 0
\end{array}\right], \quad \mathbf{K}_{j l}=\delta_{j l}\left[\begin{array}{cc}
v \mathbf{I} & 0 \\
0 & 0
\end{array}\right], \quad \mathbf{B}=\left[\begin{array}{l}
\mathbf{f} \\
0
\end{array}\right],
$$

where $k$ is an arbitrary wave number vector. Now we will compute $\mathbf{K}^{\mathrm{num}}$ from expressions $(18)-(20)$. As previously mentioned, the $\mathbf{K}_{i i}$ are singular and therefore we add a parameter $\varepsilon$ to the null diagonal term:

$$
\mathbf{K}_{i j}=\delta_{i j}\left[\begin{array}{cc}
\nu I & 0 \\
0 & \varepsilon
\end{array}\right]
$$

For the $x$-direction the eigensystem decomposition is

$$
\mathbf{K}_{x x}^{-1} A_{x}=S_{x} \Lambda_{x} S_{x}^{-1}
$$

with

$$
\mathbf{S}_{x}=\left[\begin{array}{cccc}
-\sqrt{ }(\varepsilon / v) & 0 & 0 & \sqrt{ }(\varepsilon / v) \\
0 & 1 & 0 & 0 \\
0 & 0 & 1 & 0 \\
1 & 0 & 0 & 1
\end{array}\right], \quad \mathbf{\Lambda}_{x}=\left[\begin{array}{cccc}
-\sqrt{ }(\varepsilon / v) & 0 & 0 & 0 \\
0 & 0 & 0 & 0 \\
0 & 0 & 0 & 0 \\
0 & 0 & 0 & 1 / \sqrt{ }(\varepsilon v)
\end{array}\right]
$$


$\tau_{x}$ is computed from (20) and the final expression is

$$
\tau_{x}=\operatorname{diag}\left\{\frac{h \xi}{2} \sqrt{ }\left(\frac{\varepsilon}{v}\right), \frac{h^{2}}{12 v}, \frac{h^{2}}{12 v}, \frac{h \xi}{2} \sqrt{ }\left(\frac{v}{\varepsilon}\right)\right\}
$$

where $\xi=\psi(h / \sqrt{ }(\varepsilon v))$ and $\operatorname{diag}\{a, b, c, \ldots\}$ is a diagonal matrix with diagonal entries $a, b, c, \ldots$, while for the other directions a similar calculation gives

$$
\tau_{y}=\operatorname{diag}\left\{\frac{h^{2}}{12 v}, \frac{h \xi}{2} \sqrt{\left(\frac{\varepsilon}{v}\right)}, \frac{h^{2}}{12 v}, \frac{h \xi}{2} \sqrt{ }\left(\frac{v}{\varepsilon}\right)\right\}, \quad \tau_{z}=\operatorname{diag}\left\{\frac{h^{2}}{12 v}, \frac{h^{2}}{12 v}, \frac{h \xi}{2} \sqrt{\left(\frac{\varepsilon}{v}\right)}, \frac{h \xi}{2} \sqrt{ }\left(\frac{v}{\varepsilon}\right)\right\} .
$$

The expression for $\tau$ is obtained from (19) (assuming $p=1$ for simplicity) as

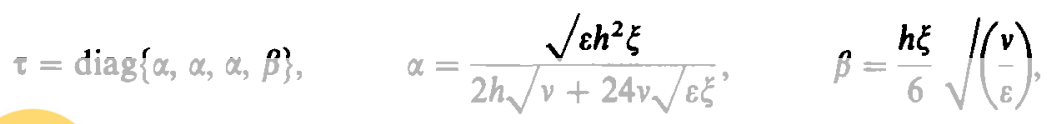

and the $\mathbf{K}_{i j}^{\text {num }}$ from expression (18) are

$$
\mathbf{K}_{i j}^{\text {num }} k_{\mathrm{i}} k_{j}=\left[\begin{array}{cc}
\beta \mathbf{k k}^{\mathrm{T}} & 0 \\
0 & \alpha k^{2}
\end{array}\right] .
$$

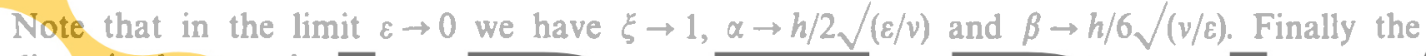
discretized system is
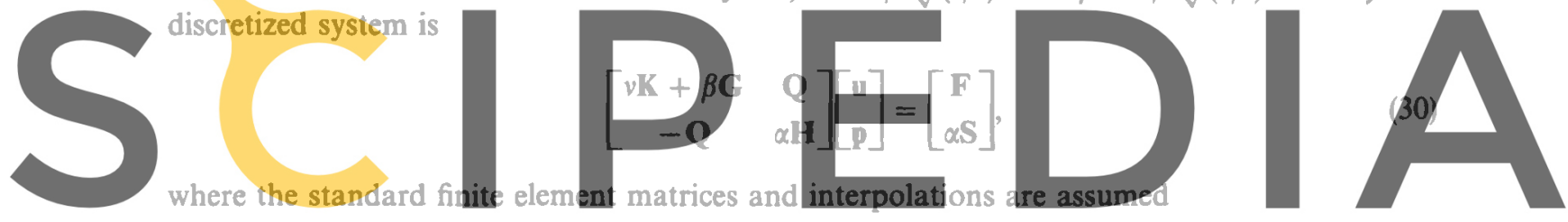

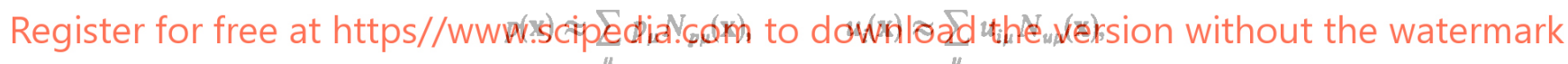

$$
\begin{aligned}
& K_{i \mu, j v}=\delta_{i j}\left(\int_{\Omega} N_{u \mu, k} N_{u v, k}\right) \mathrm{d} \Omega, \quad Q_{\mu, j v}=\int_{\Omega} N_{p \mu} N_{u v, j} \mathrm{~d} \Omega, \quad H_{\mu, v}=\int_{\Omega} N_{p \mu, k} N_{p v, k} \mathrm{~d} \Omega, \\
& G_{i \mu, j v}=\int_{\Omega} N_{u \mu, i} N_{u v, j} \mathrm{~d} \Omega, \quad F_{i \mu}=\int_{\Omega} f_{i} N_{u \mu} \mathrm{d} \Omega, \quad S_{\mu}=\int_{\Omega} f_{i} N_{p \mu, i} \mathrm{~d} \Omega .
\end{aligned}
$$

These expressions include the possibility of different interpolation spaces $V_{p}=\operatorname{span}\left\{N_{p \mu}\right\}$ and $\mathrm{V}_{u}=\operatorname{span}\left\{N_{u \mu}\right\}$ for pressure and velocity, but in fact we will always work with $\mathrm{V}_{p}=\mathrm{V}_{\mathrm{u}}$ since we are interested in equal-order interpolations.

$\mathbf{K}$ is a viscosity-rigidity matrix and $\mathbf{Q}$ and $-\mathbf{Q}^{\mathbf{T}}$ are centred discrete approximations to the divergence and gradient operators respectively. For the Galerkin formulation the operators $\mathbf{G}$ and $\mathbf{H}$ are not present and, owing to the null diagonal block in the matrix, spurious oscillations are present if the pair $\left(\mathrm{V}_{p}, \mathrm{~V}_{\mathrm{u}}\right)$ fails to satisfy the Brezzi-Babǔska condition. In practice it has been found that a necessary condition for the Brezzi-Babǔska one is that the order of interpolation for a must be one degree higher than that of $p$. Recently a great deal of research work $^{8-10,17}$ has been carried out to circumvent this restriction by means of equal-order schemes. It has been shown ${ }^{11}$ that all these formulations are based on the introduction (with different justifications varying from one author to another) of a stabilizing term proportional to the discrete version of $\Delta p$ in the continuity equation, like the term $\alpha \mathrm{H}$ in our formulation; see 
equation (30). Furthermore, as regards the term $\beta \mathbf{G}$, Sampaio's formulation is the closest to ours, since it introduces a similar term equivalent to a discrete $\nabla(\nabla \cdot u)$ in the momentum equation. This term represents physically a bulk viscosity term. It can also be found by a straightforward application of the Taylor-Galerkin method. ${ }^{11}$

Most of the stabilized methods are basically of the form (30). The case $\alpha=\beta=0$ corresponds to the Galerkin non-stabilized case. The case $\beta=0, \alpha=\alpha^{\prime} h^{2} / 2 v$ corresponds to the stabilized method of Hughes et al. ${ }^{8}$ Sampaio's scheme 9 (in the Stokes regime and for linear elements) is obtained with $\alpha=2 m h^{2} / \nu$ and $\beta=\lambda m u^{2} h^{2} / 4 v$, where $\lambda$ and $m$ are $O(1)$ constants defined in Reference 9. System (30) corresponds to the centred Galerkin discretization of the PDE system

$$
-v \Delta \mathbf{u}-\beta \nabla(\nabla \cdot \mathbf{u})+\nabla p=f, \quad \nabla \cdot \mathbf{u}-\alpha \Delta p=-\alpha(\nabla \cdot f)
$$

and can be obtained from the original Stokes system by adding $-\beta$ times the gradient of the continuity equation to the momentum equation and $-\alpha$ times the divergence of the momentum equation to the continuity equation. Since the systems are equivalent, the resulting stabilized scheme has the same precision (in the sense of truncation error order) independently of $\alpha$ and $\beta$.

\subsection{Fourier analysis of the stabilization terms}

With regard to the influence of $\alpha$ and $\beta$ on stability, we have made an in-depth discrete Fourier analysis which will not be presented in detail here, but its relevant results will. As usual, all quantities are decomposed into plane waves lik $\mu(x)=\hat{\mu v e x} \cdot x$ and response functions of the form

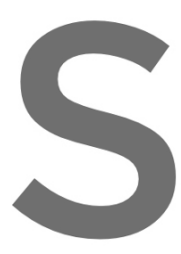
\[ \partial \hat{p} / \partial \hat{f}_{i}=G_{1}, s_{i}(k, \alpha, \beta, \ldots) \]
are computed. This last response function is very important since
for non-stable formulations at particular wave number vectors co modes':

$$
\mathbf{k}_{\text {check }}=\left\{\varepsilon_{x}, \varepsilon_{y}, \varepsilon_{z}\right\} \pi / h, \quad \varepsilon_{i}=0,1 \text {. }
$$
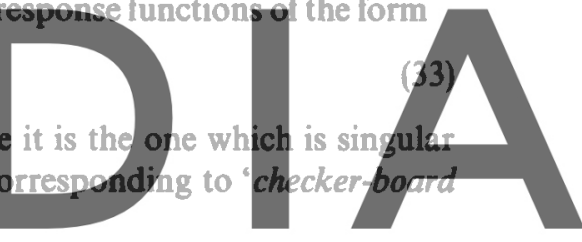

(34)

Register for free at https/ hwww.scipedia. Gom to download the version without the watermark

$-1,1,-1, \ldots\}$.

Taking $\mathbf{k}=(k, 0,0)$ for simplicity, the resulting ID discrete system is

$$
\begin{gathered}
-\alpha \frac{p_{i+1}-2 p_{i}+p_{i-1}}{h^{2}}+\frac{u_{i+1}-u_{i-1}}{2 h}=-\alpha \frac{f_{i+1}-f_{i-1}}{2 h}, \\
\frac{p_{i+1}-p_{i-1}}{2 h}-(\beta+v) \frac{u_{i+1}-2 u_{i}+u_{i-1}}{h^{2}}=\frac{f_{i+1}+4 f_{i}+f_{i-1}}{6}
\end{gathered}
$$

and the response function is

where

$$
\frac{1}{h} G_{p, f}=\frac{-i \sin (k h)\left\{\frac{1}{3}[2+\cos (k h)]+(\gamma / 4) \sin ^{2}(k h / 2)\right\}}{\gamma \sin ^{4}(k h / 2)+\sin ^{2}(k h)},
$$

$$
\gamma=16 \alpha(v+\beta) / h^{2}=16 \alpha v / h^{2}+16 \alpha \beta / h^{2}=I+I I
$$

is a global stability parameter.

In Figure 5 we can see the effect of $\gamma$ on the response function. For $\gamma>0$ the singularity is removed at $k=\pi$. However, for too small $\gamma(\gamma \ll 1)$ an undesirable peak in the response function occurs near $k=\pi$. It is seen that for $\gamma \approx 1$ the peak is completely removed and a monotonic response curve is obtained. Numerical results from Reference 8 show that for the lid-driven 


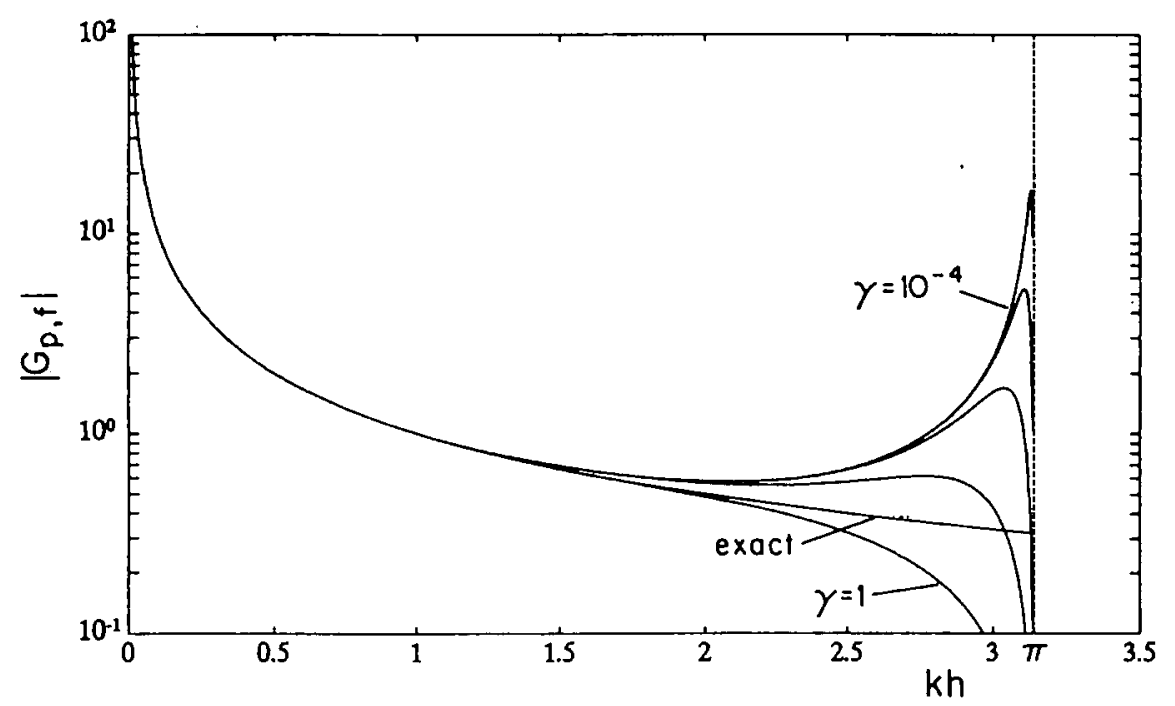

Figure 5. Response curves for several global stability parameters

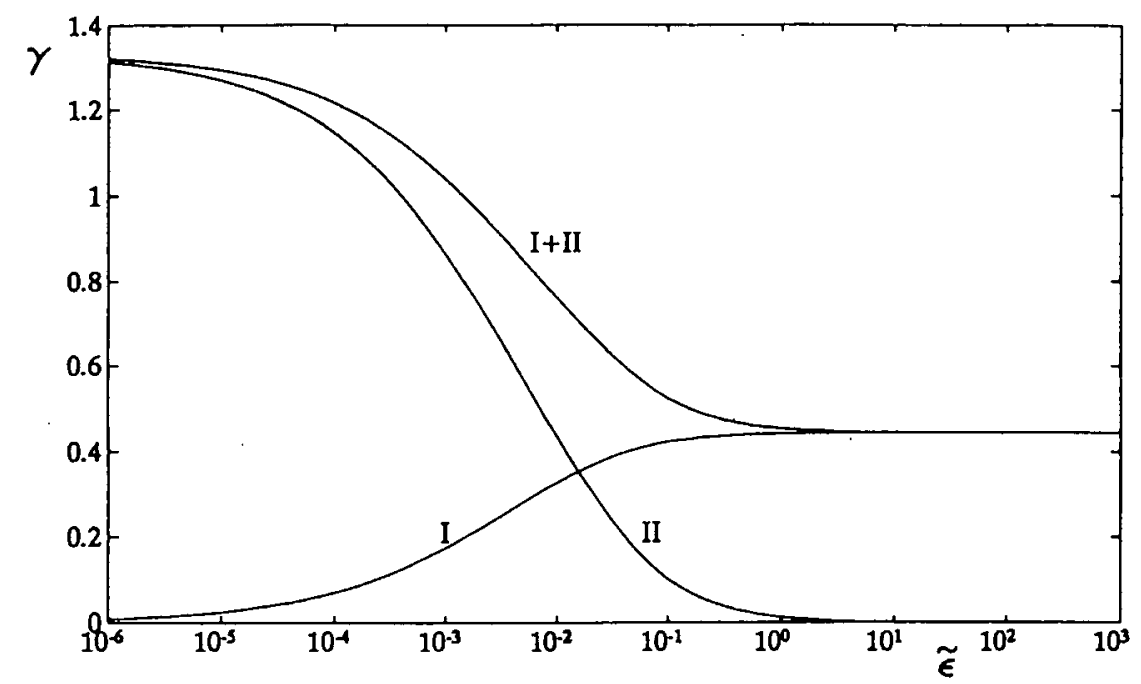

Figure 6. Global stability parameter for the proposed method

cavity flow benchmark with a global stability parameter $\gamma$ ranging from 0.8 to 8 oscillations are absent, whereas for $\gamma<0.08$ oscillations exist. These results are in perfect agreement with the previous discrete analysis.

As regards the proposed method, the global stability parameter is

$$
\gamma=\frac{4}{3} \xi \frac{\xi+6 \sqrt{\tilde{\varepsilon}}}{1+12 \sqrt{\tilde{\varepsilon} \xi}}
$$

where

$$
\tilde{\varepsilon}=\varepsilon v / h^{2}, \quad \xi=\psi(1 / \sqrt{ } \tilde{\varepsilon}),
$$


$\tilde{\varepsilon}$ being a new non-dimensional stability parameter. The relationship between $\gamma$ and $\tilde{\varepsilon}$ is depicted in Figure 6, together with each of the terms I and II in (37). We can see that irrespective of the choice of $\tilde{\varepsilon}$, the algorithm is stable $(\gamma>0.4)$. Note that for very small $\tilde{\varepsilon}$ the first term $16 \alpha v / h^{2}$ in (37) is not sufficient for stabilization but the second term $16 \alpha \beta / h^{2}$ is. Thus in this case the bulk viscosity (related to $\beta$ ) stabilization term is indispensable. Keeping the $\alpha$-term small is a nice feature, since the term $-\alpha \Delta p$ changes the nature of the system, e.g. the number of boundary conditions to be specified. Bad boundary conditions are absorbed in the boundary layers whose thickness grows with $\alpha$, a small $\alpha$ guarantees good behaviour of the scheme even if additional boundary conditions are not good. ${ }^{12}$ Note that $\beta=v / 6 \sqrt{\tilde{\varepsilon}}=O(1)$ as a function of the mesh size, but, as previously shown, this does not affect the precision.

In the numerical analysis $\tilde{\varepsilon}$ has always been chosen as $\tilde{\varepsilon}=1$.

\section{ITERATIVE SOLUTION OF THE SYSTEM OF EQUATIONS}

The spatially discretized system

$$
\mathbf{U}_{, t}=R(\mathbf{U})
$$

is solved for the steady state by iterating an explicit pseudotemporal scheme

$$
\frac{\mathbf{U}^{n+1}-\mathbf{U}^{n}}{\Delta t}=\mathbf{R}\left(\mathbf{U}^{n}\right)
$$

This kind of iterative solver becomes competitive for large 3D problems such as those found in industry. However, techniques such as local time stepping and preconditioning are needed in order to reduce the intrinsic bad conditioning due to local mesh refinements and large differences in time scales between the two phases.

\section{VALIDATION OF THE CODE}

The first validation test is the lid-driven square cavity as described in Figure 7. This test problem was selected because of the great degree of similarity with the industrial problem under

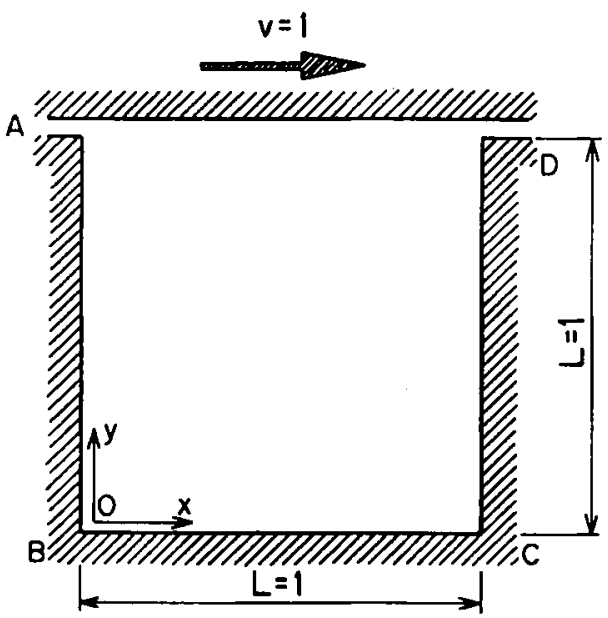

Figure 7. Lid-driven square cavity-problem description 


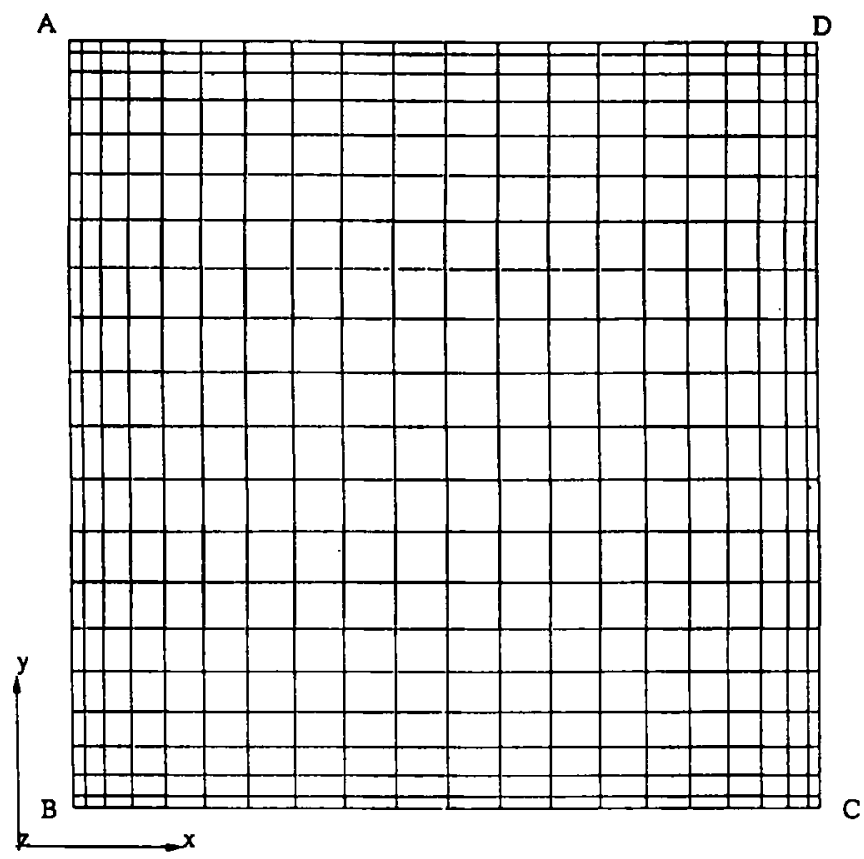

Figure 8 . Lid-driven square cavity-20 $\times 20$ non-homogeneous mesh

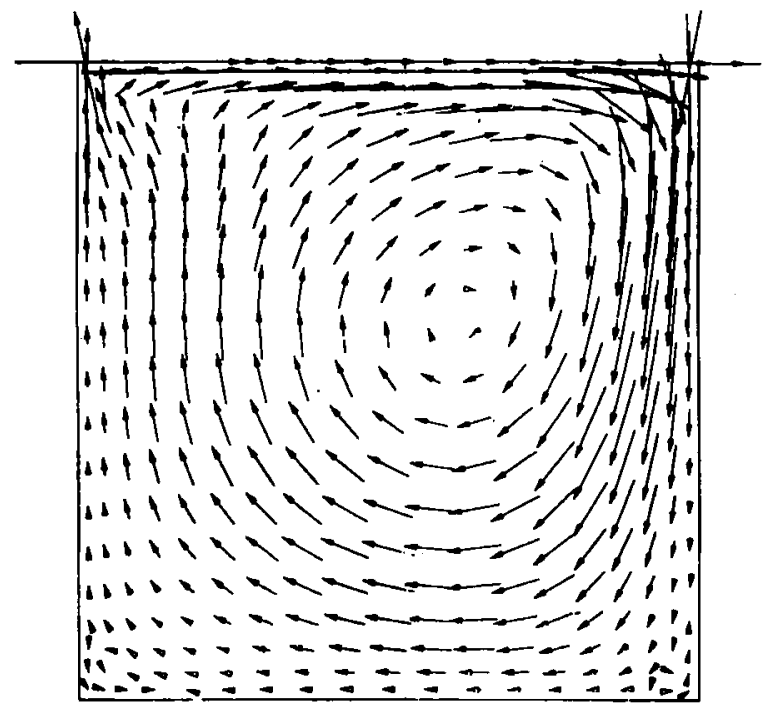

Figure 9. Lid-driven square cavity-velocity field for $R e=1000$

consideration. The FEM mesh and resulting velocity field for $R e=1000$ are shown in Figures 8 and 9 respectively, the latter being in good agreement with that obtained by other authors. ${ }^{13}$

The second test is similar but now with two solid obstacles as depicted in Figure 10. In Figure 11 the results for $R e=250$ are shown. They are in good agreement with those of Lipke and Wagner. ${ }^{14}$ 


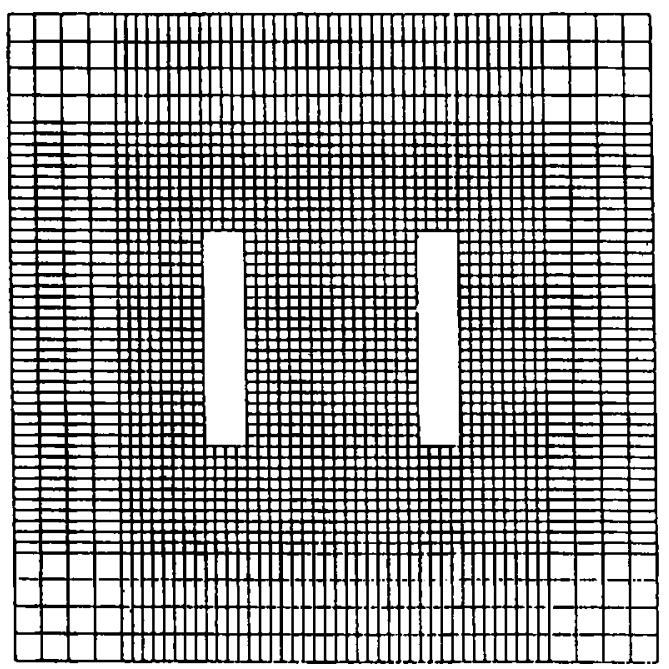

Figure 10. Multiply connected lid-driven square cavity-problem description and FEM mesh

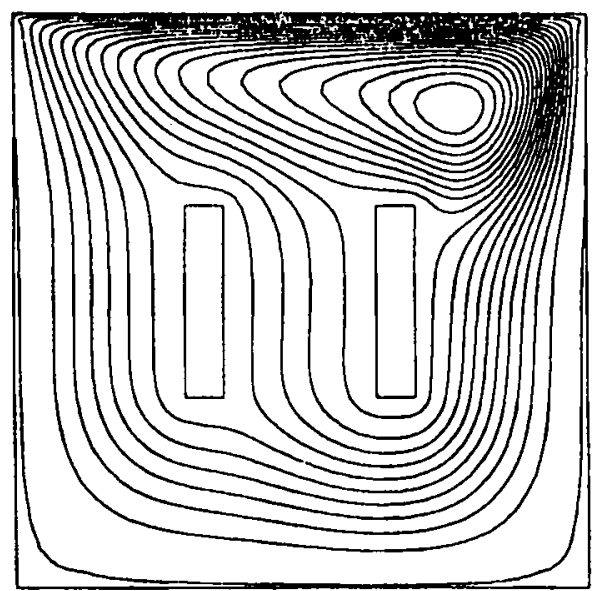

Figure 11. Multiply connected lid-driven square cavity-streamlines for $R e=\mathbf{2 5 0}$

\section{NUMERICAL RESULTS}

\subsection{Three-dimensional results}

Both the geometry and operating conditions are based on those of McKelliget et al. ${ }^{15}$ They correspond to an axisymmetric geometry, i.e. a centred inlet nozzle at the bottom of the ladle. The radius and height of the bath are $R=0.3 \mathrm{~m}$ and $H=0.6 \mathrm{~m}$ and the diameter of the inlet 
nozzle is $d_{0}=0.0127 \mathrm{~m}$. The fluids are water and air, so that $\rho=1000 \mathrm{~kg} \mathrm{~m}^{-3}$ and $\mu_{\text {tmol }}$ (molecular dynamic viscosity of water) $=1.25 \times 10^{-3} \mathrm{~kg} \mathrm{~m}^{-1} \mathrm{~s}^{-1}$. The injection velocity at the nozzle is $v_{0}=1.62 \mathrm{~m} \mathrm{~s}^{-1}$. In the same work McKelliget $e t$ al. introduce an ad. hoc turbulence model, giving an effective turbulent viscosity as a funcion of injection velocity and geometrical parameters:

$$
\mu_{\text {eff }}=\beta V_{0}\left(\rho_{\mathrm{ges}} \rho_{\mathrm{liq}}^{2} R^{2} \pi d_{0}^{2} / H\right)^{1 / 3}
$$

They show for a specific configuration that the values of the effective viscosity predicted by this simple model are very similar to those obtained by a more sophisticated two-equation one. Moreover, the predicted velocity fields are in reasonable quantitative agreement with those predicted by the more sophisticated model and also with experimental results, so we adopted the simplified model for our calculations.

For the problem at hand $\mu_{\text {eff }}=0.09 \mathrm{~kg} \mathrm{~m}^{-1} \mathrm{~s}^{-1}$. The volumetric flow rate of gas is $Q=A_{\text {nozzle }} V_{0}=2.05 \times 10^{-4} \mathrm{~m}^{3} \mathrm{~s}^{-1}$. Reynolds numbers computed from the velocities obtained by McKelliget et al. are near $5 \times 10^{6}$.

Our objective was to obtain similar results but with an essentially $3 \mathrm{D}$ geometry, i.e. with a non-centred nozzle. A 3D mesh for half the ladle was used (see Figure 12). The nozzle is placed at half the radius from the centre $T$. The gas volume fraction at the nozzle is obtained from the

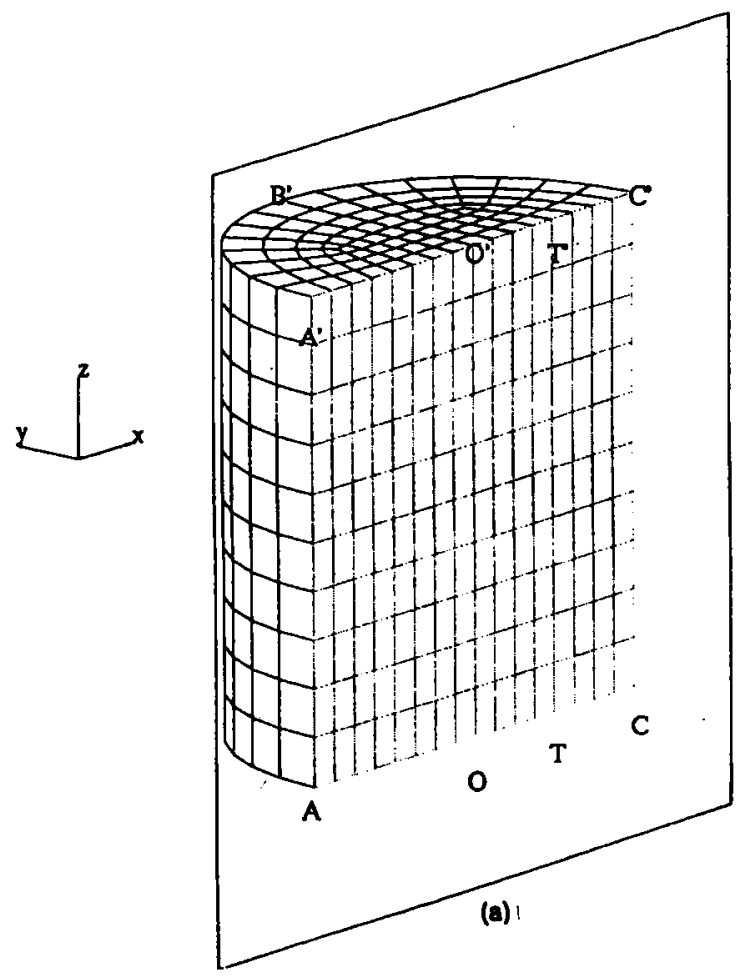

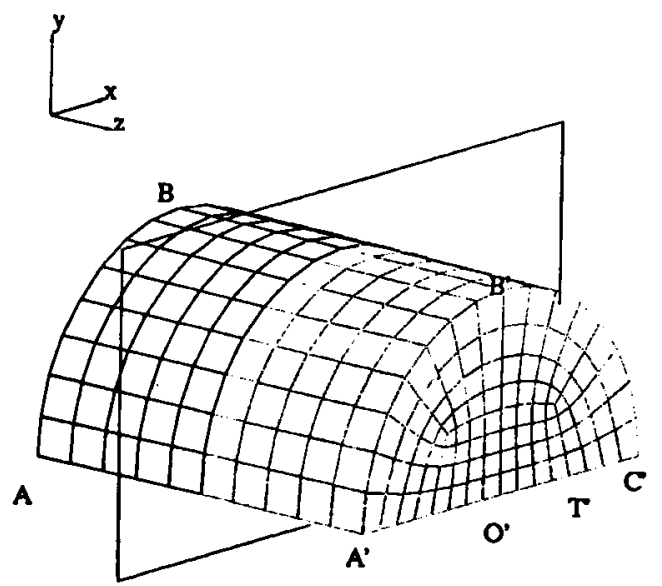

(b)

Figure 12. Cylindrical vessel with off-centre nozzle - geometry and mesh description: (a) plane cut at $y=0 \mathrm{~m}$; (b) plane cut at $z=0.3 \mathrm{~m}$ 
total volumetric flow and a simplification for the slip velocity expression at the nozzle (see equation (8)). The volumetric flow is

$$
Q=A_{\text {nozzle }}^{\alpha} U_{\text {s.nozzle }}
$$

Furthermore, at the nozzle the liquid velocity vanishes and the expression for the slip velocity allows us to obtain the gas velocity

$$
U_{\mathrm{g}, \text { nozzle }}=\frac{2}{9} r_{\mathrm{b}}^{2} \rho g / \mu_{\mathrm{mol}}(1-\alpha) .
$$

$U_{z, \text { nozzle }}$ can be eliminated from expressions (43) and (44) and an expression for the gas volume fraction at the nozzle is obtained:

$$
\alpha_{\mathrm{z}, \text { nozzle }} /\left(1-\alpha_{\mathrm{g}, \text { nozzle }}\right)=4.5 \mu_{\mathrm{mol}} Q / A_{\text {nozzle }} r_{\mathrm{b}}^{2} \rho g .
$$

We have taken a bubble radius of $r_{b}=5 \times 10^{-4} \mathrm{~m}$ at the nozzle. ${ }^{16}$ The resulting value for $\alpha$ is 0.25 .

Since Reynold numbers to be obtained are high, we made a continuation in $\alpha$ and $r_{b}$ to try to reach the operating conditions. From a theoretical analysis of the model and our experience with the code it became clear that $\alpha$ controls the driving force which turns the fluid and thus the Reynolds number to be obtained. On the other hand the bubble radius controls the interaction between the two phases through the gas velocity, which is composed of the liquid and slip velocities (equation (8)). Note that because the slip velocity is always directed upwards, the only way to deflect the gaseous bubbles from their vertical trajectory comes from the liquid velocity. The larger $r_{b}$, the higher is the slip velocity with respect to the liquid velocity. The gas is advected upwards without lateral deviation and the plume is straight. In contrast, the smaller $r_{b}$, the lower is the slip velocity. The bubbles get scattered in the fluid, being deviated by the horizontal component of the liquid velocity. The gas is redistributed and thus the friction force which drives the liquid and the coupling between the two phases are stronger.

We began with a relatively high $r_{b}$ in order to have a straight plume and study the relation between $\alpha_{\text {g,nozzle }}$ and the Reynolds number. In Figures 13 and 14 we show the results for $\alpha=1.5 \times 10^{-4}$ and $3 \times 10^{-4}$. For these values Reynolds numbers in the region of 2000 and 4000 respectively are reached. For $\alpha=1.5 \times 10^{-4}$ we obtained results for $r_{b}=2.8 \times 10^{-4}$ and $10^{-4}$. In Figure 15 we show isocurves for the gaseous volume fraction, where the influence of this parameter on the expansion of the gaseous plume is evident.

Actually, the mesh used in this example is rather coarse, because we have strong limitations on CPU speed. In spite of this fact, the numerical results are admissible in an industrial context.

\subsection{Two-dimensional results}

The geometry and mesh are shown in Figure 16(a). The geometry is a rectangle of height $\mathrm{BB}^{\prime}=H=0.6 \mathrm{~m}$ and width $2 \mathrm{~TB}=2 R=0.6 \mathrm{~m}$. The nozzle is at the centre $\mathrm{T}$ and, owing to the symmetry of the problem, only the right-half part TBB'T'T is considered. The nozzle width is $0.015 \mathrm{~m}$ (equivalent to three elements). To calculate the area of the nozzle, we assume a characteristic length $z$ of $\pi R / 2$, so that the total volume of the vessel is $H=H \times 2 R \times \pi R / 2=$ $\pi R^{2} H$, equal to that of McKelliget et al. ${ }^{15}$

In Figure 16(b) we show the velocity pattern for $Q=1.36 \times 10^{-5} \mathrm{~m}^{3} \mathrm{~s}^{-1}$. Note that the rotation centre is much higher than in the $3 \mathrm{D}$ runs where $R e$ was much lower. The expansion of the hydrodynamic jet is clearly seen, a feature which was poorly resolved in the $3 \mathrm{D}$ case owing to the coarseness of the grid. 

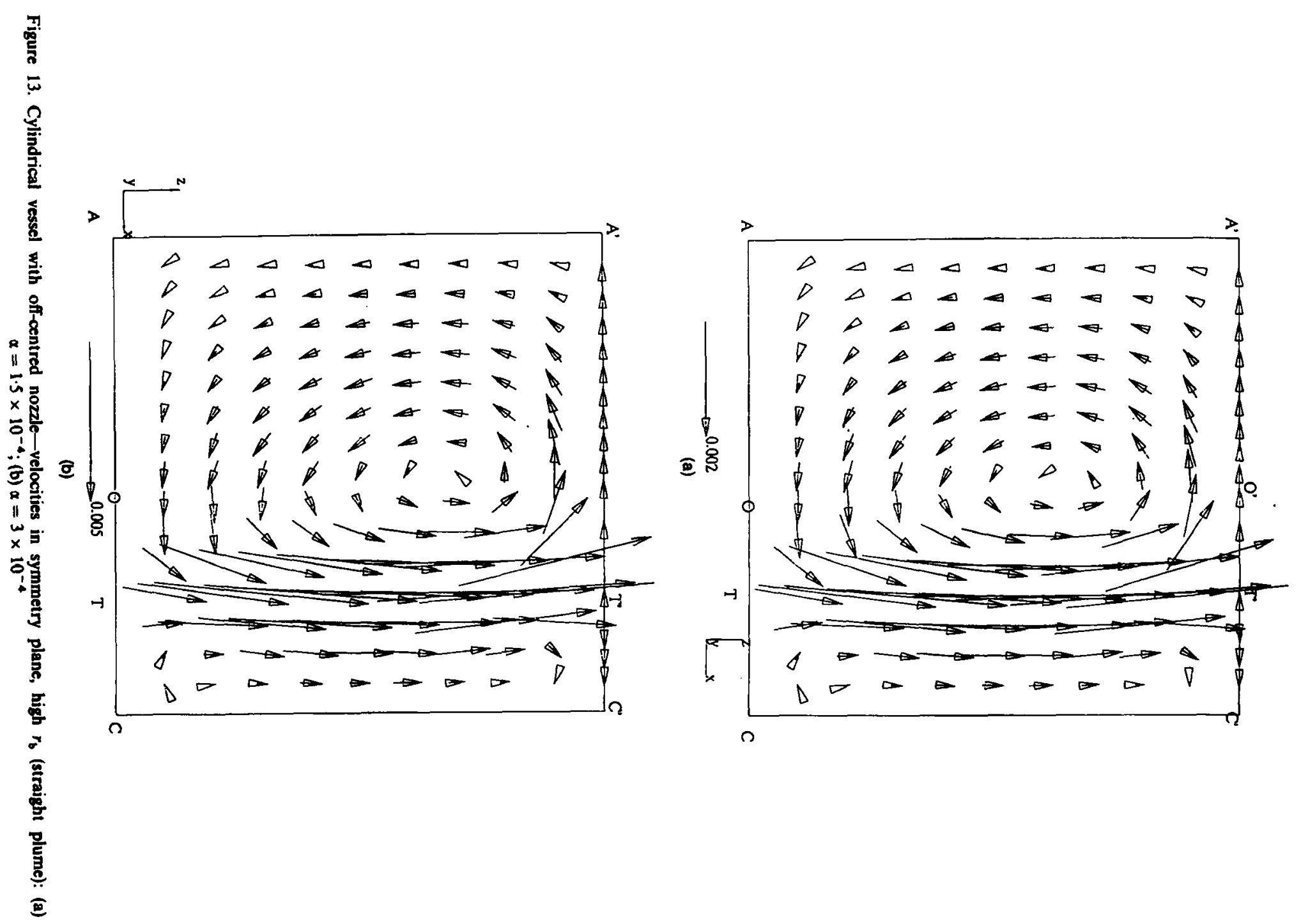


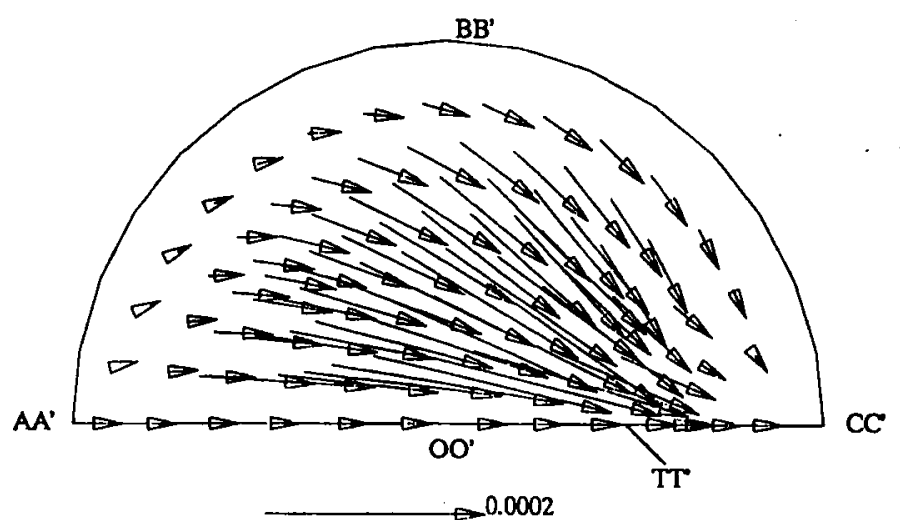

(a)

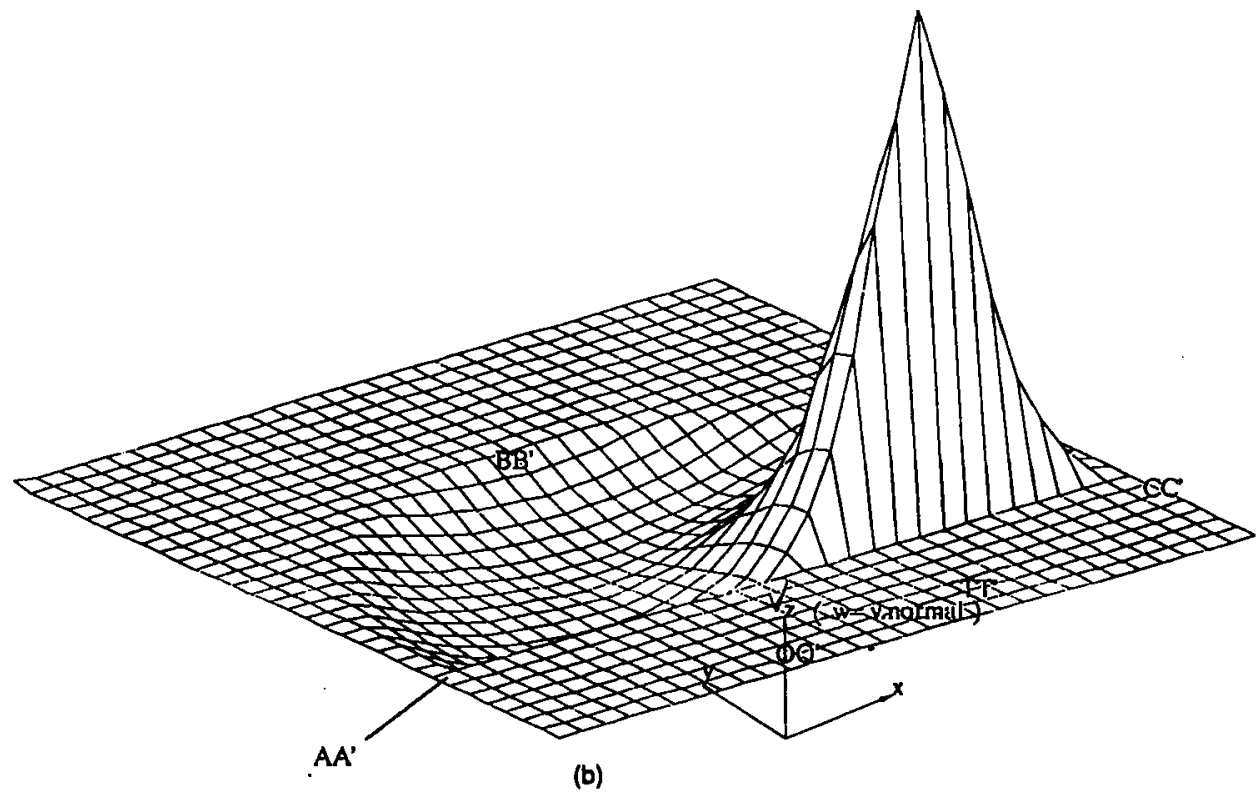

Figure 14. Cylindrical vessel with off-centre nozzle-velocities in $z=0.3 \mathrm{~m}$ plane, high $r_{\mathrm{b}}$ (straight plume), $\alpha=1.5$ $\times 10^{-4}$ : (a) in-plane velocities; (b) 3D view of normal velocities 
FLOW MODELLING IN GAS-STIRRED VESSELS

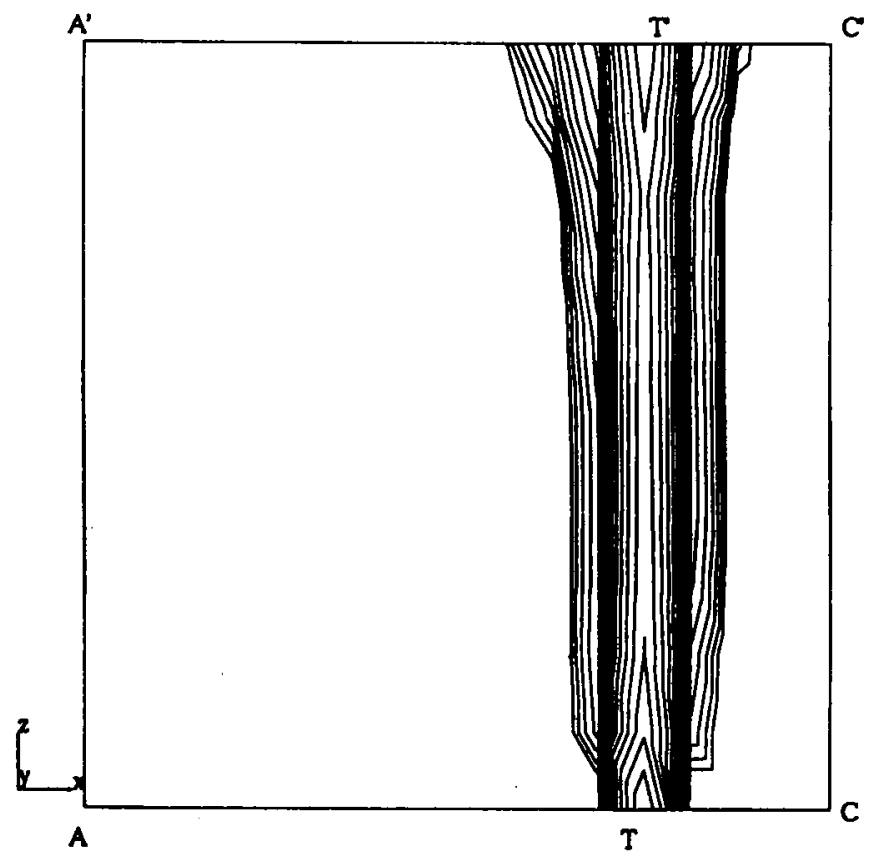

(a)!

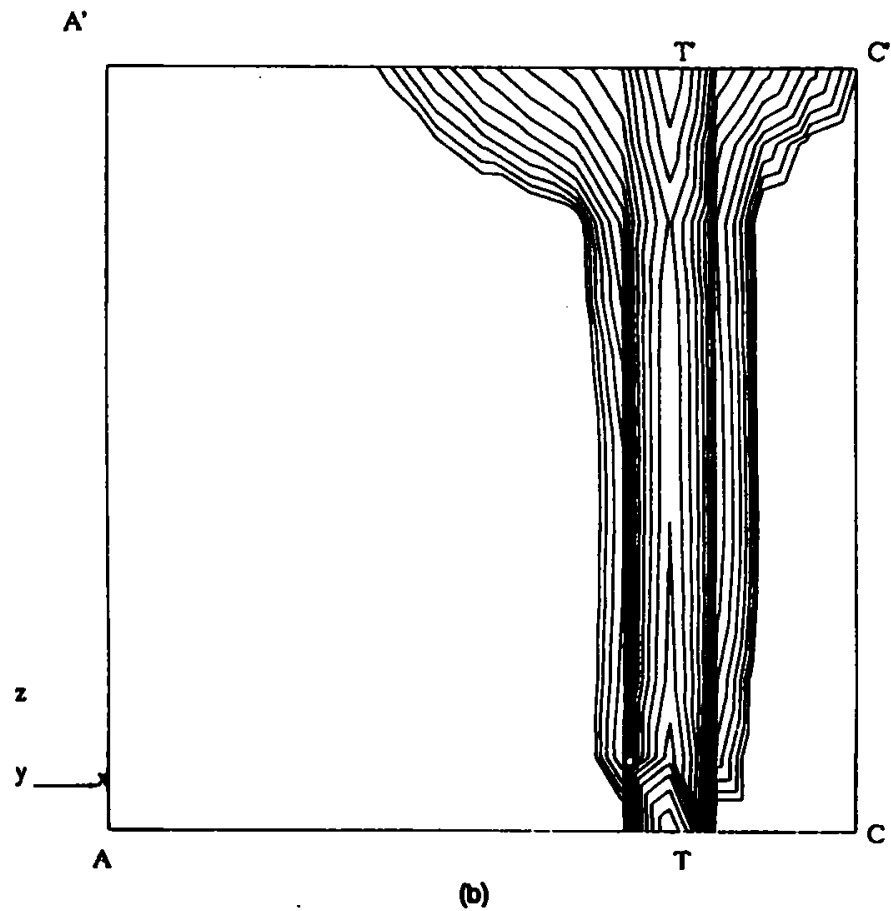

Figure 15. Cylindrical veseel with off-centre nozzle - gas volume fraction isocurves at symmetry plane $y=0 \mathrm{~m}$, $\alpha=1.5 \times 10^{-4}:$ (a) $r_{b}=2.8 \times 10^{-4} ;$ (b) $r_{b}=10^{-4}$ 


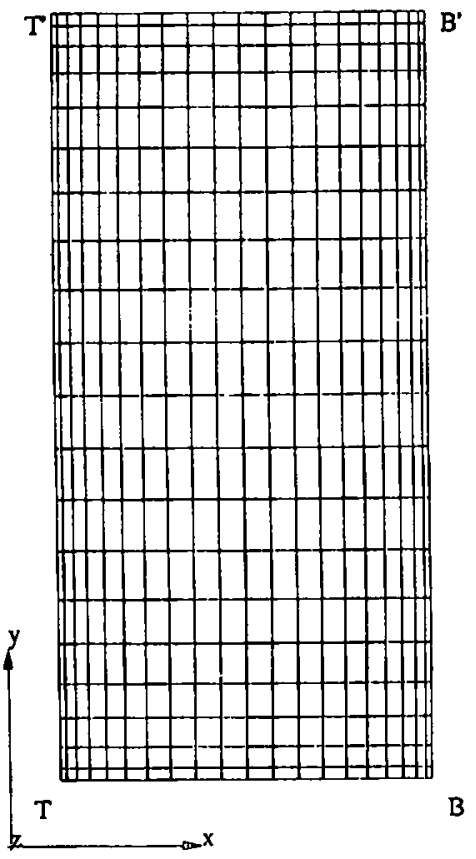

(a)

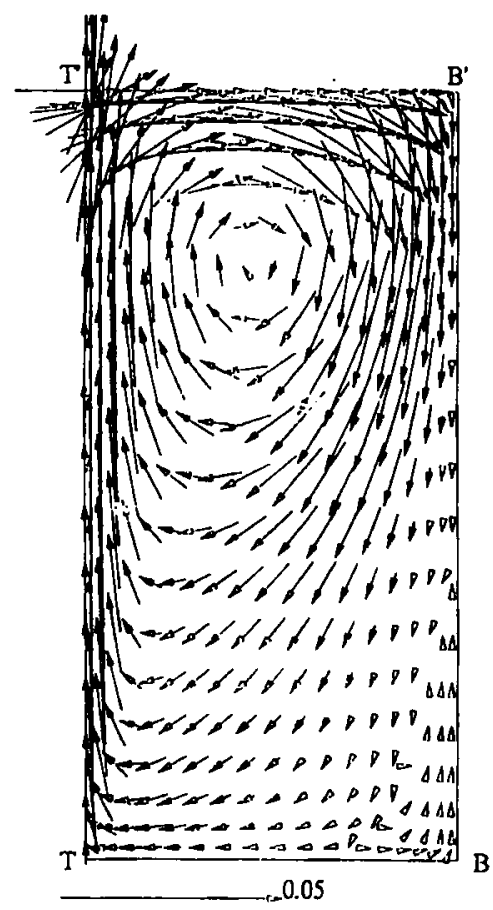

(b)

Figure 16. Plane geometry simulation: (a) geometry and FEM mesh; (b) velocity vectors

\section{CONCLUSIONS}

We have presented a two-phase Navier-Stokes 3D code based on equal-order interpolation and explicit temporal integration. This kind of algorithm is more efficient in CPU time and memory requirements. A simple calculation shows that the amount of memory required to store a non-symmetric matrix for a cube of $10 \times 10 \times 10$ linear elements (close to the number of elements in our discretization), with four degrees of freedom per node, is roughly $24 \mathrm{Mb}$. All our runs were made in a system with $4 \mathrm{Mb}$ RAM and 0.5 Mflops CPU performance.

The Navier-Stokes solver has been validated with the square lid-driven cavity test problem. Several promising numerical results for typical gas-stirred vessels were obtained. Attention is focused on the fluid mechanical part of the problem. We consider that an improved turbulence model is a key feature for future work.

\section{ACKNOWLEDGEMENT}

The authors wish to express their gratitude to Consejo Nacional de Investigaciones Científicas y Técnicas (CONICET, Argentina) for its financial support.

\section{APPENDIX: NOMENCLATURE}

$\mathbf{X}, \mathbf{X}$ vectors in lowercase, matrices in uppercase

$X_{, i} \partial X / \partial x_{i}$ 


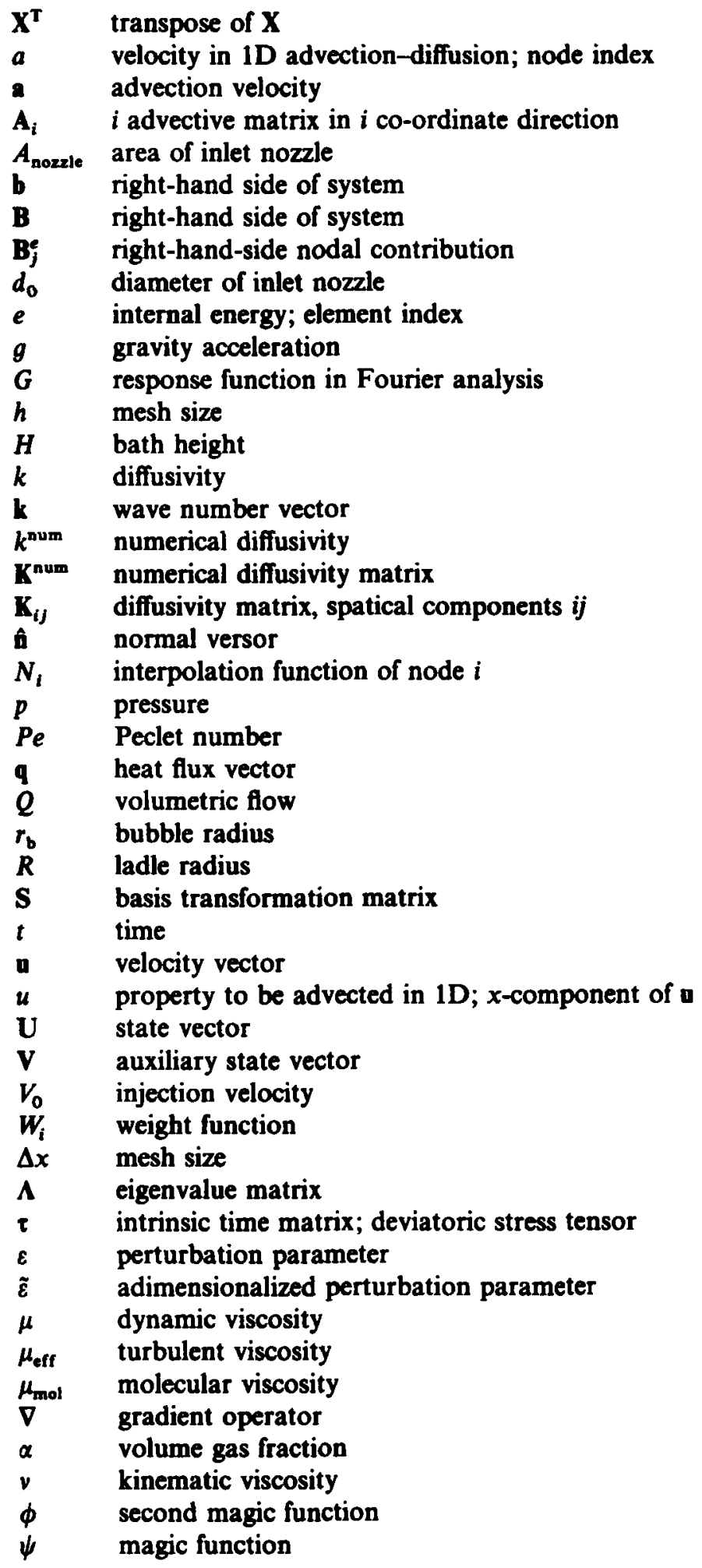




$\begin{array}{ll}\tau & \text { scalar intrinsic time } \\ \Delta & \text { Laplace operator } \\ \Delta x & \text { grid spacing } \\ \lambda_{m} & \text { eigenvalue } \\ \rho & \text { density } \\ \Omega & \text { volume }\end{array}$

\section{REFERENCES}

1. J. M. Delhaye, M. Giot and M. L. Riethmuller (eds), Thermohydraulics of Two-phase Systems for Inchustrial Design and Nuclear Engineering, Hemisphere, Washington, DC, 1981.

2. M. Ishii, Thermo-fuid Dynamic Theory of Two-phase Flow, Eyrolles, Paris, 1975.

3. A. D. Drew and R. T. Lahey, 'Application of general constitutive principles to the derivation of multidimensional two-phase flow equations', Int. J. Multiphase Flow, 5, 243-264 (1979).

4. A. D. Drew, L. Cheng and R. T. Lahey, 'The analysis of virtual mass effects in two phase flow', Int. J. Multiphase Flow, 5, 233-242 (1979).

5. L. D. Landau and E. M. Lifshitz, Fluid Mechanics, Pergamon, Oxford, 1959.

6. T. J. R. Hughes, M. Mallet and A. Mizukami, 'A new finite element formulation for CFD: II. Beyond SUPG', Comput. Methods Appl. Mech. Eng., 54, 341-355 (1986).

7. M. Mallet, 'A finite element method for CFD', Ph.D. Thesis, Stanford University, 1985.

8. T. J. R. Hughes, L. Franca and M. Balestra, 'A new finite element formulation for CFD: V. Circumventing the Babüska-Brezzi condition: a stable Petrov-Galerkin formulation of the Stokes problem accommodating equal-order interpolations', Comput. Methods Appl. Mech. Eng., 59, 85-99 (1986).

9. S. L. Frey, L. P. Franca and R. Sampaio, 'Stabilized methods for the incompressible Navier-Stokes flow', in H. Alder et al. (eds), Proc. Int. Congr. on Numerical Methods in Engineering and Applied Sciences, Concepción, November 1992.

10. O. C. Zienkiewicz, J. Szmelter and J. Peraire, 'Compressible and incompressible flow: an algorithm for all seasons', Comput. Methods Appl. Mech. Eng., 78, 105-121 (1990).

11. O. C. Zienkiewicz and J. Wu, 'Incompressibility without tears-how to avoid restrictions on mixed formulations', Int. j. numer. methods eng., 32, 1189-1204 (1991-1992).

12. M. Storti, 'Discrete Fourier analysis of Stokes flow', GTM Internal Rep. I-12/92, 1992.

13. R. Schreiber and H. B. Keller, 'Driven-cavity flows by efficient numerical techniques', J. Comput. Phys., 49, 310-333 (1983).

14. A. Lipke and H. Wagner, 'Numerical solution of the Navier-Stokes equations in multiply domains connected', Comput. Fluids, 20, 19-27 (1991).

15. J. W. McKelliget, M. Cross and R. D. Gibson, 'A turbulent fluid flow model of gas-agitated reactors', Appl. Math. Modell., 6, 469-480 (1982).

16. K. Y. M. Lai and M. Salcudean, 'Computer analysis of multidimensional, turbulent, buoyancy-induced, two-phase flows in gas-agitated-liquid reactors', Comput. Fluids, 15, 281-295 (1987).

17. T. J. R. Hughes, L. Franca and M. Balestra, 'A new finite element formulation for CFD: VII. The Stokes problem with various well-posed boundary conditions: symmetric formulations that converge for all velocity/pressure spaces', Comput. Methods Appl. Mech. Eng., 65, 85-96 (1987). 